
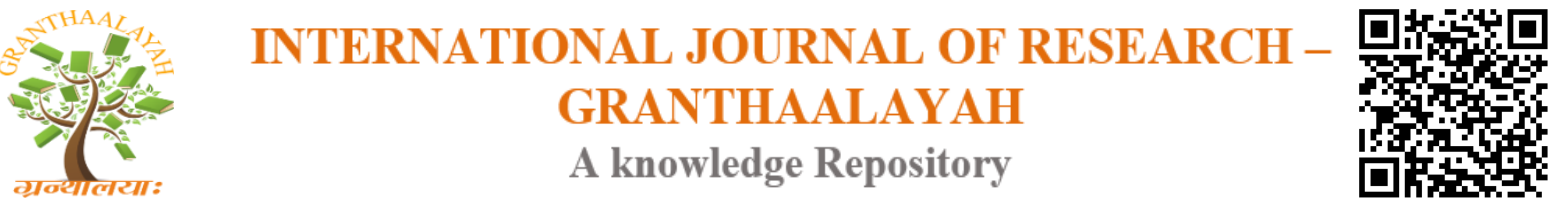

Science

\title{
TAILORED PARTICLE SWARM OPTIMIZATION ALGORITHM FOR SOLVING OPTIMAL REACTIVE POWER PROBLEM
}

\author{
Dr.K.Lenin ${ }^{* 1}$ \\ ${ }^{* 1}$ Professor, Department of EEE, Prasad V.Potluri Siddhartha Institute of Technology, Kanuru, \\ Vijayawada, Andhra Pradesh -520007, India
}

\begin{abstract}
This paper presents Tailored Particle Swarm Optimization (TPSO) algorithm for solving optimal reactive power problem. Particle Swarm optimization algorithm based on Membrane Computing is proposed to solve the problem. Tailored Particle Swarm Optimization (TPSO) algorithm designed with the framework and rules of a cell-like P systems, and particle swarm optimization with the neighbourhood search. In order to evaluate the efficiency of the proposed algorithm, it has been tested on standard IEEE 118 \& practical 191 bus test systems and compared to other specified algorithms. Simulation results show that Tailored Particle Swarm Optimization (TPSO) algorithm is superior to other algorithms in reducing the real power loss.
\end{abstract}

Keywords: Membrane Computing; Particle Swarm Optimization; Optimal Reactive Power; Transmission Loss.

Cite This Article: Dr.K.Lenin. (2017). "TAILORED PARTICLE SWARM OPTIMIZATION ALGORITHM FOR SOLVING OPTIMAL REACTIVE POWER PROBLEM." International Journal of Research - Granthaalayah, 5(12), 246-255.

\section{Introduction}

The main objective of optimal reactive power problem is to minimize the real power loss and bus voltage deviation. Various numerical methods like the gradient method [1-2], Newton method [3] and linear programming [4-7] have been adopted to solve the optimal reactive power dispatch problem. Both the gradient and Newton methods have the complexity in managing inequality constraints. If linear programming is applied then the input- output function has to be uttered as a set of linear functions which mostly lead to loss of accuracy. The problem of voltage stability and collapse play a major role in power system planning and operation [8]. Evolutionary algorithms such as genetic algorithm have been already proposed to solve the reactive power flow problem [9-11]. Evolutionary algorithm is a heuristic approach used for minimization problems by utilizing nonlinear and non-differentiable continuous space functions. In [12], Hybrid differential evolution algorithm is proposed to improve the voltage stability index. In [13] Biogeography Based algorithm is projected to solve the reactive power dispatch problem. In [14], a fuzzy based method is used to solve the optimal reactive power scheduling method. In 
[15], an improved evolutionary programming is used to solve the optimal reactive power dispatch problem. In [16], the optimal reactive power flow problem is solved by integrating a genetic algorithm with a nonlinear interior point method. In [17], a pattern algorithm is used to solve ac-dc optimal reactive power flow model with the generator capability limits. In [18], F. Capitanescu proposes a two-step approach to evaluate Reactive power reserves with respect to operating constraints and voltage stability. In [19], a programming based approach is used to solve the optimal reactive power dispatch problem. In [20], A. Kargarian et al present a probabilistic algorithm for optimal reactive power provision in hybrid electricity markets with uncertain loads. This paper presents Tailored Particle Swarm Optimization (TPSO) algorithm for solving optimal reactive power problem. Particle Swarm optimization algorithm [21] based on Membrane Computing is proposed to solve the problem. Membrane computing (P systems) was initiated by Paun [22] in 1998, which is a category of new-fangled computing replica abstracted from the structure and functioning of living cells, as well as from the interactions of living cells in tissues $[23,24]$. In recent years, many variant of membrane computing models have been developed rapidly, and also have turned out that membrane computing has an important probable to be applied for variety of computationally hard problems. In order to evaluate the efficiency of the proposed algorithm, it has been tested on standard IEEE 118 \& practical 191 bus test systems and compared to other specified algorithms. Simulation results show that Tailored Particle Swarm Optimization (TPSO) algorithm is superior to other algorithms in reducing the real power loss.

\section{Problem Formulation}

\subsection{Active Power Loss}

The objective of the reactive power problem is to minimize the active power loss in the transmission network, which can be described as follows:

$F=P L=\sum_{k \in N b r} g_{k}\left(V_{i}^{2}+V_{j}^{2}-2 V_{i} V_{j} \cos \theta_{i j}\right)$

Or

$F=P L=\sum_{i \in N g} P_{g i}-P_{d}=P_{g s l a c k}+\sum_{i \neq s l a c k}^{N g} P_{g i}-P_{d}$

Where $g_{k}$ : is the conductance of branch between nodes $\mathrm{i}$ and $\mathrm{j}$, Nbr: is the total number of transmission lines in power systems. $\mathrm{P}_{\mathrm{d}}$ : is the total active power demand, $\mathrm{P}_{\mathrm{gi}}$ : is the generator active power of unit $i$, and $\mathrm{P}_{\text {gsalck: }}$ is the generator active power of slack bus.

\subsection{Voltage Profile Improvement}

For minimizing the voltage deviation in PQ buses, the objective function becomes:

$F=P L+\omega_{v} \times V D$

Where $\omega_{\mathrm{v}}$ : is a weighting factor of voltage deviation. 
VD is the voltage deviation given by:

$V D=\sum_{i=1}^{N p q}\left|V_{i}-1\right|$

\subsection{Equality Constraint}

The equality constraint of the problem is represented by the power balance equation, where the total power generation must cover the total power demand and the power losses,

$P_{G}=P_{D}+P_{L}$

This equation is solved by running Newton Raphson load flow method, by calculating the active power of slack bus to determine active power loss.

\subsection{Inequality Constraints}

The inequality constraints reflect the limits on components in the power system as well as the limits created to ensure system security. Upper and lower bounds on the active power of slack bus, and reactive power of generators:

$P_{\text {gslack }}^{\min } \leq P_{\text {gslack }} \leq P_{\text {gslack }}^{\max }$

$Q_{g i}^{\min } \leq Q_{g i} \leq Q_{g i}^{\max }, i \in N_{g}$

Upper and lower bounds on the bus voltage magnitudes:

$V_{i}^{\min } \leq V_{i} \leq V_{i}^{\max }, i \in N$

Upper and lower bounds on the transformers tap ratios:

$T_{i}^{\min } \leq T_{i} \leq T_{i}^{\max }, i \in N_{T}$

Upper and lower bounds on the compensators reactive powers:

$Q_{c}^{\min } \leq Q_{c} \leq Q_{C}^{\max }, i \in N_{C}$

Where $\mathrm{N}$ is the total number of buses, $\mathrm{N}_{\mathrm{T}}$ is the total number of Transformers; $\mathrm{N}_{\mathrm{c}}$ is the total number of shunt reactive compensators.

\section{Particle Swarm Optimization Algorithm Based on Membrane Computing}

Particle Swarm optimization algorithm (PSO) based on Membrane Computing is proposed to solve the problem. Tailored Particle Swarm Optimization (TPSO) algorithm designed with the framework and rules of a cell-like $\mathrm{P}$ systems, and particle swarm optimization with the neighbourhood search. 


\subsection{Cell-Like P Systems}

$\mathrm{P}$ systems can be classified into the cell-like P systems, the tissue-like P systems and neural-like $\mathrm{P}$ systems. In cell-like $\mathrm{P}$ systems, the covering structure is a hierarchical arrangement of membranes embedded in the skin membrane. A membrane without any other membranes within is assumed to be an elementary membrane. Every membrane has an area containing a multi-set of objects and a set of evolutionary rules. The multi-sets of objects progress in each region and move from a region to a neighbouring one by applying the rules in a nondeterministic and maximally similar way.

The membrane structure of a cell-like P system can be formally defined as follows,

$\Pi=\left(o . T, u, s_{1}, \ldots, s_{n}, R_{1}, ., R_{n}, i_{0}\right)$

Where;

(i) $O$ is the alphabet of objects;

(ii) $T$ is the output alphabet, $T \subseteq O$;

(iii) $\mu$ is a membrane structure consisting of $n$ membranes, and the membranes labelled with 1 , $2, . ., n ; n$ is called the degree of the system $\Pi$;

(iv) $s_{i}(1 \leq i \leq n)$ are strings which representmulti-sets over $O$ associated with the region $1,2, .$. , $n$ of $\mu$.

(v) $R_{i}(1 \leq i \leq n)$ are the evolution rules over $O^{*}, R_{i}$ is associated with region $i$ of $\mu$, and it is of the following forms.

(a) $\left[i s_{1} \rightarrow s_{2}\right]$, where $i \in\{1,2, . ., n\}$, and $s_{1}, s_{2} \in o^{*}$

(Evolution rules: a rule of this type works on astring objects by the local search algorithm or various evolutionary operators, and the new strings object are created in region $i$.)

(b) $s_{1}[i] \rightarrow\left[i s_{2}\right]_{i}$, Where $i \in\{1,2, . ., n\}$, and $s_{1}, s_{2} \in o^{*}$.

(Send-in communication rules; a string object is transmitted in the region $i$.)

(c) $\left[i s_{1}\right]_{i} \rightarrow[i]_{i} s_{2}$, where $i \in\{1,2, . ., n\}$, and

$S_{1}, S_{2} \in O^{*}$.

(Send-out communication rules; a string objectis sent out of the region $i$.)

(vi) $i_{o}$ is the output membrane.

A "P" system, regarded as a model of computation, and is called as membrane algorithm, which is poised of a series of computing steps between configurations. Each calculation starts from the primary configuration, and halts when there are no more rules applicable in anyregion. In the computing procedure, the system will go from one configuration to a new one by applying the regulations related to regions in a non-deterministic and maximally equivalent manner. The result of the calculation is obtained in region $i_{o}$.

The membrane algorithm

Start

Initialize the membrane structure and parameters, gen $=0$; 
While (Not termination condition) do

Evaluate the evolution rules in all elementary membranes;

Determine the fitness by the fitness function;

Perform the communication rules;

Record the current best solution;

gen $=$ gen +1 ;

end while

End start

\subsection{Standard PSO}

Particle Swarm optimization algorithm (PSO) is a population-based, co-operative search metaheuristic introduced by Kennedy and Eberhart. The fundament for the development of PSO is hypothesis that a potential solution to an optimization problem is treated as a bird without quality and volume, which is called a particle, coexisting and evolving simultaneously based on knowledge sharing with neighbouring particles. While flying through the problem search space, each particle modifies its velocity to find a better solution (position) by applying its own flying experience (i.e. memory having best position found in the earlier flights) and experience of neighbouring particles (i.e. best-found solution of the population). Particles update their positions and velocities as shown below:

$v_{t+1}^{i}=\omega_{t} \cdot v_{t}^{i}+c_{1} \cdot R_{1} \cdot\left(p_{t}^{i}-x_{t}^{i}\right)+c_{2} \cdot R_{2} \cdot\left(p_{t}^{g}-x_{t}^{i}\right)$

$x_{t+1}^{i}=x_{t}^{i}+v_{t+1}^{i}$

Where $x_{t}^{i}$ represents the current position of particle $\mathrm{i}$ in solution space and subscript $\mathrm{t}$ indicates an iteration count; $p_{t}^{i}$ is the best-found position of particle $\mathrm{i}$ up to iteration count $\mathrm{t}$ and represents the cognitive contribution to the search velocity $v_{t}^{i}$. Each component of $v_{t}^{i}$ can be clamped to the range to control excessive roaming of particles outside the search space; $p_{t}^{g}$ is the global bestfound position among all particles in the swarm up to iteration count $t$ and forms the social contribution to the velocity vector; $r_{1}$ and $r_{2}$ are random numbers uniformly distributed in the interval $(0,1)$, where $c_{1}$ and $c_{2}$ are the cognitive and social scaling parameters, respectively; $\omega_{t}$ is the particle inertia, which is reduced dynamically to decrease the search area in a gradual fashion . The variable $\omega_{t}$ is updated as

$\omega_{t}=\left(\omega_{\max }-\omega_{\min }\right) \cdot \frac{\left(t_{\max }-t\right)}{t_{\max }}+\omega_{\min }$

Where $\omega_{\max }$ and $\omega_{\min }$ denote the maximum and minimum of $\omega_{t}$ respectively; $t_{\max }$ is a given number of maximum iterations. Particle i fly toward a new position according to Eq. (12) and (13). In this way, all particles of the swarm find their new positions and apply these new positions to update their individual best $p_{t}^{i}$ points and global best $p_{t}^{g}$ of the swarm. This process is repeated until termination conditions are met. In the paper, the velocity equation of PSO is modified as follows. 
$v_{(t+1)}^{i}=\left|r_{1}()\right| \times\left(\operatorname{Pbest}_{i j}(t)-x_{i j}(t)\right)+\left|r_{2}()\right| \times\left(\operatorname{gbest}_{j}(t)-x_{i j}-x_{i j}(t)\right)$

The procedure of Particle Swarm optimization algorithm based on Membrane Computing for solving optimal reactive power dispatch problem is described as follows.

Step 1: Initialize membrane structure and $X(t)$,

$V(t)$ and Multi-sets are initialized as follows:

$s_{0}=\lambda$

$s_{1}=b_{1,1} b_{1,2}, \ldots, b_{1, m}$

$\ldots$

$s_{n}=b_{n, 1} b_{n, 2}, \ldots, b_{n, m}$

Step 2: progress rules in each of the region 1 to $n$ are implemented. The particle swarm optimization (PSO) based on Gaussian distribution will be carry out in every elementary membrane concurrently.

Step 3: Implement the send-out communication rules, the strings are sent to skin membrane from each elementary membrane.

Step 4: To improve the disadvantage of the premature convergence problem, the local and global neighbourhood searches are implemented in the skin membrane to improve the ability of exploration and exploitation. The equations of local neighbourhood search are defined as follows

$L X_{i}=r_{1} \cdot X_{i}+r_{2} \cdot$ pbest $_{i}+r_{3}\left(X_{c}-X_{d}\right)$

$L V_{i}=V_{i}$

Where $X i$ is the position vector of the $i$-th particle, pbest $t_{i}$ is the previous best particle of $P_{i} ; X_{c}$ and $X_{d}$ are the position vectors of two random particles.c, $d \in[i+k, i-k]$.

The equations of global search are shown as follows,

$G X_{i}=r_{4} \cdot X_{i}+r_{5} \cdot$ pbest $_{i}+r_{6}\left(X_{e}-X_{f}\right)$

$G V_{i}=V_{i}$

Where gbest is the global best particle, $X_{e}$ and $X_{f}$ are the position vectors of two random particles chosen from the entire swarm, $e, f \in[1, N] . r_{4}, r_{5}$ and $r_{6}$ are three uniform random numbers in $[0$, 1],

Step 5: Determine the fitness of each string object by fitness function, and save the existing best strings;

Step 6: Execute the send-in communication rules between the skin membrane and each elementary membrane concurrently. The detail explanation is as follows. 
(i) First, the best strings and $m-1$ strings with the worst fitness are sent to the elementary membrane1;

(ii) Subsequently, in the remaining strings, the current best strings and $m$ - 1 strings with the worst fitness are sent to the elementary membrane 2 ;

(iii) The above process is executed constantly until the strings from the skin membrane back to each region;

Step 7: If the stopping condition is met, then out put the results; otherwise, return to step 2.

\section{Simulation Results}

At first Tailored Particle Swarm Optimization (TPSO) algorithm has been tested in standard IEEE 118-bus test system [25].The system has 54 generator buses, 64 load buses, 186 branches and 9 of them are with the tap setting transformers. The limits of voltage on generator buses are $0.95-1.1$ per-unit., and on load buses are $0.95-1.05$ per-unit. The limit of transformer rate is 0.9 -1.1 , with the changes step of 0.025 . The limitations of reactive power source are listed in Table 1 , with the change in step of 0.01 .

Table 1: Limitation of reactive power sources

\begin{tabular}{|l|l|l|l|l|l|l|l|}
\hline BUS & 5 & 34 & 37 & 44 & 45 & 46 & 48 \\
\hline QCMAX & 0 & 14 & 0 & 10 & 10 & 10 & 15 \\
\hline QCMIN & -40 & 0 & -25 & 0 & 0 & 0 & 0 \\
\hline BUS & 74 & 79 & 82 & 83 & 105 & 107 & 110 \\
\hline QCMAX & 12 & 20 & 20 & 10 & 20 & 6 & 6 \\
\hline QCMIN & 0 & 0 & 0 & 0 & 0 & 0 & 0 \\
\hline
\end{tabular}

The statistical comparison results of 50 trial runs have been list in Table 2 and the results clearly show the better performance of proposed Tailored Particle Swarm Optimization (TPSO) algorithm.

Table 2: Comparison results

\begin{tabular}{|l|l|l|l|l|}
\hline Active power loss (p.u) & $\begin{array}{l}\text { BBO } \\
{[\mathbf{2 6}]}\end{array}$ & $\begin{array}{l}\text { ILSBBO/ } \\
\text { strategy1 } \\
{[\mathbf{2 6}]}\end{array}$ & $\begin{array}{l}\text { ILSBBO/ } \\
\text { strategy1 } \\
{[\mathbf{2 6}]}\end{array}$ & $\begin{array}{l}\text { Proposed } \\
\text { TPSO }\end{array}$ \\
\hline Min & 128.77 & 126.98 & 124.78 & 108.02 \\
\hline Max & 132.64 & 137.34 & 132.39 & 114.54 \\
\hline Average & 130.21 & 130.37 & 129.22 & 110.36 \\
\hline
\end{tabular}

Then the Tailored Particle Swarm Optimization (TPSO) algorithm has been tested in practical 191 test system and the following results have been obtained. In Practical 191 test bus system Number of Generators $=20$, Number of lines $=200$, Number of buses $=191$ Number of transmission lines $=55$. Table 3 shows the optimal control values of practical 191 test system obtained by TPSO method. And table 4 shows the results about the value of the real power loss by obtained by Tailored Particle Swarm Optimization (TPSO) algorithm. 
Table 3: Optimal Control values of Practical 191 utility (Indian) system by TPSO method

\begin{tabular}{|l|l|l|l|l|}
\hline VG1 & 1.100 & & VG 11 & 0.900 \\
\hline VG 2 & 0.760 & VG 12 & 1.000 \\
\hline VG 3 & 1.010 & VG 13 & 1.000 \\
\hline VG 4 & 1.010 & VG 14 & 0.900 \\
\hline VG 5 & 1.100 & VG 15 & 1.000 \\
\hline VG 6 & 1.100 & VG 16 & 1.000 \\
\hline VG 7 & 1.100 & VG 17 & 0.900 \\
\hline VG 8 & 1.010 & VG 18 & 1.000 \\
\hline VG 9 & 1.100 & VG 19 & 1.100 \\
\hline VG 10 & 1.010 & VG 20 & 1.100 \\
\hline
\end{tabular}

\begin{tabular}{|c|c|c|c|c|c|}
\hline $\mathrm{T} 1$ & 1.000 & $\mathrm{~T} 21$ & 0.900 & T41 & 0.900 \\
\hline $\mathrm{T} 2$ & 1.000 & T22 & 0.900 & T42 & 0.900 \\
\hline T3 & 1.000 & $\mathrm{~T} 23$ & 0.900 & T43 & 0.910 \\
\hline $\mathrm{T} 4$ & 1.100 & T24 & 0.900 & T44 & 0.910 \\
\hline T5 & 1.000 & $\mathrm{~T} 25$ & 0.900 & T45 & 0.910 \\
\hline T6 & 1.000 & T26 & 1.000 & T46 & 0.900 \\
\hline $\mathrm{T} 7$ & 1.000 & T27 & 0.900 & T47 & 0.910 \\
\hline $\mathrm{T} 8$ & 1.010 & $\mathrm{~T} 28$ & 0.900 & $\mathrm{~T} 48$ & 1.000 \\
\hline T9 & 1.000 & T29 & 1.010 & T49 & 0.900 \\
\hline T10 & 1.000 & T30 & 0.900 & $\mathrm{~T} 50$ & 0.900 \\
\hline $\mathrm{T} 11$ & 0.900 & T31 & 0.900 & T51 & 0.900 \\
\hline $\mathrm{T} 12$ & 1.000 & T32 & 0.900 & T52 & 0.900 \\
\hline T13 & 1.010 & T33 & 1.010 & T53 & 1.000 \\
\hline $\mathrm{T} 14$ & 1.010 & T34 & 0.900 & T54 & 0.900 \\
\hline T15 & 1.010 & T35 & 0.900 & T55 & 0.900 \\
\hline T19 & 1.020 & T39 & 0.900 & & \\
\hline T20 & 1.010 & T40 & 0.900 & & \\
\hline
\end{tabular}

Table 4: Optimum real power loss values obtained for practical 191 utility (Indian) system by TPSO method

\begin{tabular}{|l|l|}
\hline Real power Loss (MW) & TPSO \\
\hline Min & 132.014 \\
\hline Max & 138.002 \\
\hline Average & 136.078 \\
\hline
\end{tabular}

\section{Conclusion}

In this paper Tailored Particle Swarm Optimization (TPSO) algorithm successfully solved optimal reactive power problem. Particle Swarm optimization algorithm based on Membrane Computing is proposed to solve the problem. Tailored Particle Swarm Optimization (TPSO) algorithm designed with the framework and rules of a cell-like P systems, and particle swarm optimization with the neighbourhood search. In order to evaluate the efficiency of the proposed algorithm, it has been tested on standard IEEE 118 \& practical 191 bus test systems and 
compared to other specified algorithms. Simulation results show that Tailored Particle Swarm Optimization (TPSO) algorithm is superior to other algorithms in reducing the real power loss.

\section{References}

[1] O.Alsac, B. Scott, "Optimal load flow with steady state security”, IEEE Transaction. PAS -1973, pp. 745-751.

[2] Lee K Y ,Paru Y M , Oritz J L -A united approach to optimal real and reactive power dispatch , IEEE Transactions on power Apparatus and systems 1985: PAS-104 : 1147-1153

[3] A.Monticelli , M .V.F Pereira ,and S. Granville, "Security constrained optimal power flow with post contingency corrective rescheduling", IEEE Transactions on Power Systems :PWRS-2, No. 1, pp.175-182., 1987.

[4] Deeb N, Shahidehpur S.M, Linear reactive power optimization in a large power network using the decomposition approach. IEEE Transactions on power system 1990: 5(2) : 428-435

[5] E. Hobson ,'Network consrained reactive power control using linear programming, ' IEEE Transactions on power systems PAS -99 (4) ,pp 868=877, 1980

[6] K.Y Lee, Y.M Park, and J.L Oritz, "Fuel -cost optimization for both real and reactive power dispatches", IEE Proc; 131C,(3), pp.85-93.

[7] M.K. Mangoli, and K.Y. Lee, "Optimal real and reactive power control using linear programming", Electr.Power Syst.Res, Vol.26, pp.1-10,1993.

[8] C.A. Canizares, A.C.Z.de Souza and V.H. Quintana, "Comparison of performance indices for detection of proximity to voltage collapse," vol. 11. no.3, pp.1441-1450, Aug 1996.

[9] K.Anburaja, "Optimal power flow using refined genetic algorithm", Electr.Power Compon.Syst , Vol. 30, 1055-1063,2002.

[10] D. Devaraj, and B. Yeganarayana, "Genetic algorithm based optimal power flow for security enhancement", IEE proc-Generation.Transmission and. Distribution; 152, 6 November 2005.

[11] A. Berizzi, C. Bovo, M. Merlo, and M. Delfanti, "A ga approach tocompare orpf objective functions including secondary voltage regulation,"Electric Power Systems Research, vol. 84, no. 1, pp. $187-194,2012$.

[12] C.-F. Yang, G. G. Lai, C.-H. Lee, C.-T. Su, and G. W. Chang, "Optimalsetting of reactive compensation devices with an improved voltagestability index for voltage stability enhancement," International Journalof Electrical Power and Energy Systems, vol. 37, no. 1, pp. $50-57,2012$.

[13] P. Roy, S. Ghoshal, and S. Thakur, "Optimal var control for improvementsin voltage profiles and for real power loss minimization usingbiogeography based optimization," International Journal of ElectricalPower and Energy Systems, vol. 43, no. 1, pp. 830 - 838, 2012.

[14] B. Venkatesh, G. Sadasivam, and M. Khan, "A new optimal reactivepower scheduling method for loss minimization and voltage stabilitymargin maximization using successive multi-objective fuzzy lp technique,'IEEE Transactions on Power Systems, vol. 15, no. 2, pp. 844 -851, may 2000.

[15] W. Yan, S. Lu, and D. Yu, "A novel optimal reactive power dispatchmethod based on an improved hybrid evolutionary programming technique,"IEEE Transactions on Power Systems, vol. 19, no. 2, pp. $913-918$, may 2004.

[16] W. Yan, F. Liu, C. Chung, and K. Wong, "A hybrid genetic algorithminteriorpoint method for optimal reactive power flow," IEEE Transactionson Power Systems, vol. 21, no. 3, pp. 1163 1169, aug. 2006.

[17] J. Yu, W. Yan, W. Li, C. Chung, and K. Wong, "An unfixed piecewiseoptimalreactive powerflow model and its algorithm for ac-dc systems," IEEE Transactions on Power Systems, vol. 23, no. 1 , pp. $170-176$, feb.2008. 
[18] F. Capitanescu, "Assessing reactive power reserves with respect tooperating constraints and voltage stability," IEEE Transactions on PowerSystems, vol. 26, no. 4, pp. 2224-2234, nov. 2011.

[19] Z. Hu, X. Wang, and G. Taylor, "Stochastic optimal reactive powerdispatch: Formulation and solution method," International Journal ofElectrical Power and Energy Systems, vol. 32, no. 6, pp. $615-621,2010$.

[20] A. Kargarian, M. Raoofat, and M. Mohammadi, "Probabilistic reactivepower procurement in hybrid electricity markets with uncertain loads,"Electric Power Systems Research, vol. 82, no. 1, pp. 68 - 80, 2012.

[21] B. Zhao, C. X. Guo, and Y. J. Cao, "A multiagent based particle swarm optimization approach for reactive power dispatch,” IEEE Trans. Power Syst., vol. 20, no. 2, pp. 1070-1078, May 2005.

[22] G. H. Paun. "Computing with Membranes". Technical Report. Finland: Turku Center for Computer Science, 1998.

[23] J. H. Xiao, X. Y. Zhang, J. Xu. "A membrane evolutionary algorithm for DNA sequence design in DNA computing". Chinese Science Bulletin, 2012, 57(6): 698-706.

[24] G. Paun. "Tracing some open problems in membrane computing". Romanian Journal of Information Science and Technology, 2007, 10: 303-314.

[25] IEEE, "The IEEE 30-bus test system and the IEEE 118-test system", (1993), http://www.ee.washington.edu/trsearch/pstca/.

[26] Jiangtao Cao, Fuli Wang and Ping Li, "An Improved Biogeography-based Optimization Algorithm for Optimal Reactive Power Flow", International Journal of Control and Automation Vol.7, No.3 (2014), pp.161-176.

*Corresponding author.

E-mail address: gklenin@ gmail.com 